\title{
Triangle-Free Planar Graphs as Segments Intersection Graphs
}

\author{
N. de Castro ${ }^{1}$, F. J. Cobos ${ }^{1}$, J.C. Dana ${ }^{1}$, A. Márquez ${ }^{1}$, and M. Noy ${ }^{2}$ \\ 1 Departamento de Matemática Aplicada I \\ Universidad de Sevilla, Spain \\ \{natalia, cobos, dana, almar\}@cica.es \\ 2 Dpto. de Matemática Aplicada II \\ Universitat Politècnica de Catalunya $^{\star \star \star}$, Spain \\ noy@grec.upc.es
}

\begin{abstract}
We prove that every triangle-free planar graph is the graph of intersection of a set of segments in the plane. Moreover, the segments can be chosen in only three directions (horizontal, vertical and oblique) and in such a way that no two segments cross, i.e., intersect in a common interior point.
\end{abstract}

\section{Introduction}

Given a set $S$ of segments in the plane, its intersection graph has a vertex for every segment and two vertices are adjacent if the corresponding segments intersect. Intersection graphs of segments and other geometrical objects have been widely studied in the past.

For instance, if the segments are contained in a straight line then we have the interval graphs [4, a well-known family of perfect graphs. If the segments are chords of a circle then the intersection graph is called a circle graph, see for instance [68].

In the general case, there is no satisfactory characterization, but some results are known for planar graphs. The most interesting one is due to de Fraysseix, Osona de Mendez and Pach [3], and independently to Ben-Arroyo Hartman, Newman and Ziv [2], which says that every planar bipartite graph is the intersection graph of a set of horizontal and vertical segments (on the other hand, it is known that the recognition of such graphs is an NP-complete problem [5]).

This result provides a partial answer to a question of Scheinerman [7]: is every planar graph the intersection graph of a set of segments in the plane?

The main result in this paper, which is a significant extension of [3], is that every triangle-free planar graph is the intersection graph of a family of segments. Moreover, the segments can be drawn in only three directions and in such a way that they do not cross. This particular class of intersection graphs is also known as contact graphs. We call such a representation a segment representation of the graph.

* Partially supported by DGES-MEC-PB96-0005-C02 
A key point in our proof is Grötszch's Theorem [9, which guarantees that every planar triangle-free graph is 3-colorable. The sketch of the proof is as follows. Given a triangle-free plane graph $G$, adding new vertices and (induced) paths between the vertices of $G$ we can obtain a new triangle-free plane graph which is a subdivision of a 3-connected graph. Starting with a 3-coloring, a segment representation in three directions is obtained for this new graph using several technical lemmas. Finally, removing the dummy vertices and paths, we obtain a segment representation of the graph $G$. The three directions considered are horizontal, vertical and oblique (parallel to the bisector of the second quadrant of the plane).
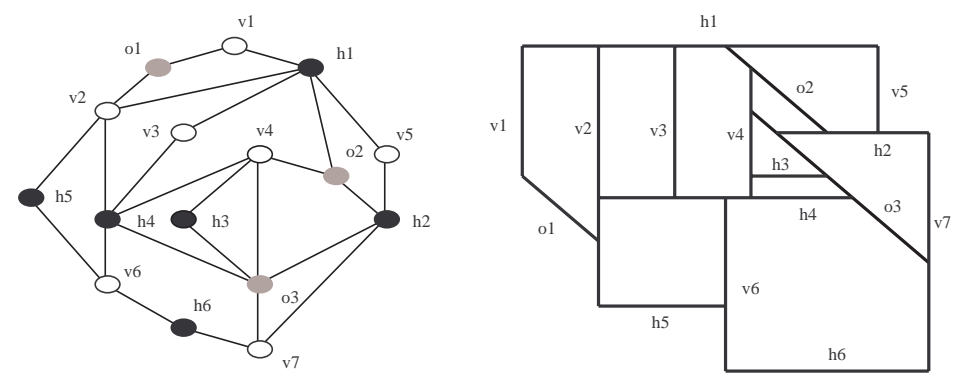

Fig. 1. A segment representation of a planar graph.

\section{Convex Faces with Three Directions}

Let $I_{G}$ be a segment representation of a plane graph $G$. A segment path of length $n$ is a sequence of segments $P=\left\{s_{1}, \ldots, s_{n}\right\}$ such that segment $s_{i}$ is adjacent to $s_{i-1}$ and $s_{i+1}$ for $1<i<n$. The representation $I_{G}$ divides the plane into regions that we call faces of the representation and which correspond to the faces of $G$. A face $F$ of a segment representation is convex if its boundary can be divided into four paths (clockwise) $P_{1}, P_{2}, P_{3}$ and $P_{4}$ such that if $s$ is a segment with extremes in the points $\left(x_{1}, y_{1}\right)$ and $\left(x_{2}, y_{2}\right)$ then:

1. if $s$ is in $P_{1}$, then $x_{1}<x_{2}$ if $s$ is horizontal, $y_{1}>y_{2}$ if $s$ is vertical and $x_{1}<x_{2}$ and $y_{1}>y_{2}$ if $s$ is oblique;

2. if $s$ is in $P_{2}$, then $x_{1}>x_{2}$ if $s$ is horizontal, $y_{1}>y_{2}$ if $s$ is vertical and $x_{1}>x_{2}$ and $y_{1}<y_{2}$ if $s$ is oblique;

3. if $s$ is in $P_{3}$, then $x_{1}>x_{2}$ if $s$ is horizontal, $y_{1}<y_{2}$ if $s$ is vertical and $x_{1}>x_{2}$ and $y_{1}<y_{2}$ if $s$ is oblique;

4. if $s$ is in $P_{4}$, then $x_{1}<x_{2}$ if $s$ is horizontal, $y_{1}<y_{2}$ if $s$ is vertical and $x_{1}<x_{2}$ and $y_{1}>y_{2}$ if $s$ is oblique. 
It can be seen that the partition of a convex face into $P_{1}, P_{2}, P_{3}$ and $P_{4}$ is not unique, for instance in the example of Figure 2 the first segment of $P_{1}$ could be in $P_{4}$. If the segments $s_{1}, \ldots, s_{n}$ are the intersection between two convex faces $F$ and $G$, we will assign those segments to only one path in each face, following the newt rule: if this segments belongs to $P_{1}$ in $F$, then they belongs to $P_{3}$ in $G$ and reciprocally. Analogously if they belongs to $P_{2}$ in $F$, then they are in $P_{4}$ of $G$ and reciprocally. This rule alow us to fix the partition of a convex face into the four paths depending on the adjacent faces if the partition is not unique.

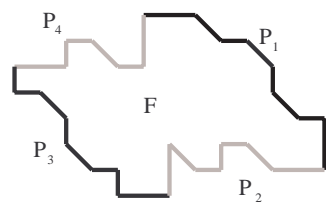

Convex Face

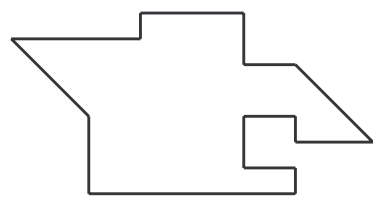

Non Convex Face

Fig. 2. The four paths of a convex face.

Lemma 1. Let $I_{G}$ be a segment representation of a plane graph $G$ such that all its faces are convex, let $k$ be a positive real number and let $s$ be a segment of length $l$ in a face $F$ of $I_{G}$. Then, $I_{G}$ can be transformed into another segment representation $I_{G}^{\prime}$ satisfying:

1. $I_{G}$ and $I_{G}^{\prime}$ have the same faces and face boundaries as $G$, and the faces of $I_{G}^{\prime}$ are convex;

2. the length of $s$ in $I_{G}^{\prime}$ is $k+l$;

3. at most two segments of $F$ (other than s) have different length in $I_{G}$ and $I_{G}^{\prime}$.

Proof. Let $P_{1}, P_{2}, P_{3}$ and $P_{4}$ denote the paths which make $F$ convex. Call the upper part (the lower part) of the face the union of the paths $P_{4}$ and $P_{1}\left(P_{2}\right.$ and $P_{3}$, respectively). Similarly, the right part (the left part) is the union of the paths $P_{1}$ and $P_{2}\left(P_{3}\right.$ and $P_{4}$, respectively).

The proof is by induction on the number of faces of the graph $G$. Suppose that $I_{G}$ is just the cycle $F$ and let $s$ be the segment of $F$ we want to enlarge. Without loss of generality we can suppose that $s$ is a horizontal segment.

If $s$ is in the upper part of $F$, we seek another horizontal segment $s^{\prime}$ in the lower part of $F$. If there exists such a segment, we enlarge $s$ and $s^{\prime}$ by the same amount and we make a translation of all the segments between $s$ and $s^{\prime}$, transforming $F$ into other convex face (see Figure 3). Otherwise, there must exist a vertical segment $s^{\prime}$ and an oblique segment $s^{\prime \prime}$ in the lower part of $F$, because the boundary of $F$ is a closed path. In this case, we increase the length of $s, s^{\prime}$ and $s^{\prime \prime}$ and we make a translation of the rest of the segments (see Figure 3). 
According to the above remark, we suppose in the rest of the proof that there exists a segment parallel to $s$ in the opposite part of $F$.

Suppose now that $I_{G}$ has two convex faces $F$ and $F^{\prime}$ sharing the segments $s_{1}, \ldots, s_{n}$. First of all, by the convexity of $F$ and $F^{\prime}$, observe that if $s_{1}, \ldots, s_{n}$ are segments of $P_{1}\left(P_{2}\right)$, then $s_{1}, \ldots, s_{n}$ form part of $P_{3}^{\prime}\left(P_{4}^{\prime}\right.$, respectively). Thus, suppose that $s_{1}, \ldots, s_{n}$ belong to $P_{1}$ in $F$ and to $P_{3}^{\prime}$ in $F^{\prime}$. If $s$ is in the upper part of $F$, we seek another horizontal segment $s^{\prime}$ in the lower part of $F$ and we proceed as before, increasing the length of $s$ and $s^{\prime}$.
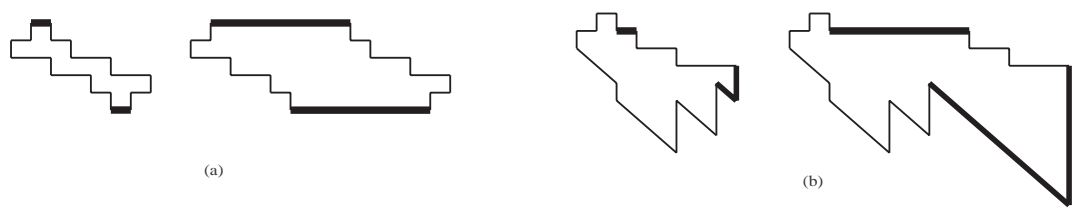

Fig. 3. How to enlarge a segment of a convex face by any amount, (a) using parallel segments or (b) using three segments.

Obviously, if $s$ is not one of the $s_{i}$, the transformation in $F$ does not affect the segments of $F^{\prime}$. But, if $s$ is one of the $s_{i}$ then, on account of the first case, we consider a horizontal segment $s^{\prime}$ in the lower part of $F$ and a horizontal segment $s^{\prime \prime}$ in the upper part of $F^{\prime}$. Increasing the length of $s, s^{\prime}$ and $s^{\prime \prime}$, and making a translation of all the segments of $F$ between $s$ and $s^{\prime}$, and all the segments of $F^{\prime}$ between $s$ and $s^{\prime \prime}$, we obtain a new segment representation with the same convex faces as $I_{G}$.

Suppose now that $I_{G}$ has $n$ convex faces, $F_{1}, \ldots, F_{n}$, and let $s$ be a segment of a face $F \neq F_{n}$, where $F_{n}$ lies in the outer face of $I_{G}$, i.e., the vertices $v_{1}, \ldots, v_{k}$ of $G$, corresponding to the segments $s_{1}, \ldots, s_{k}$ of $F_{n}$, lie on the outer face, and $v_{2}, \ldots, v_{k-1}$ are of degree two. Let us consider the graph $G_{1}=G-\left\{v_{2}, \ldots, v_{k-1}\right\}$. Removing the segments of $I_{G}$ corresponding to $v_{2}, \ldots, v_{k-1}$, we obtain a segment representation $I_{G_{1}}$ of $G_{1}$.

Since $I_{G_{1}}$ has $n-1$ faces, we can transform its segment representation into another representation which has the same convex faces, enlarging in any amount some of the segments. To obtain this new representation, we have to enlarge other segments in $I_{G_{1}}$ besides $s$, but we have preserved the structure of the segments in the representation, so we are able to represent again the segments corresponding to $v_{2}, \ldots, v_{k-1}$ enlarging the length of one (or two) of them if necessary, obtaining a segment representation of $G$. 


\section{Triangle-Free Graphs}

Using the results of Barnette in [1], it can be deduced easily that every planar triangle-free graph which is a subdivision of a 3-connected graph can be reduced, by deleting edges and paths with internal vertices of degree two, to a subdivision of the complete graph $K_{4}$ in such a way that in each step we have a subdivision of a 3 -connected graph. Moreover, if we fix a subgraph of $G$ which is a subdivision of $K_{4}, G$ can be reduced, by deleting edges and paths, to this subgraph. This result will allow us to build a segment representation in three directions of subdivisions of 3-connected graphs as follows. Firstly we build a segment representation of a subdivision of $K_{4}$ and then insert, in reverse order, the edges and paths that were removed to obtain from $G$ the subdivision of $K_{4}$.

In order to do this, we need two basic operations: (1) insert a path between two segments of the same face and (2) join two segments of the same face. The second operation is, at least, as difficult as the first one, so we will concentrate only in the second operation.

We can see, using Lemma 1, that by enlarging some segments, a segment representation with convex faces can be transformed into another one preserving the topology of the embedding. But this is not sufficient to carry on the second operation, so we need another kind of transformation. Fixing an index $i$ and changing the drawing of the segments of the path $P_{i}$ of a face $F$, it is possible to draw a new convex face $F^{\prime}$ where the segments that were in $P_{i}$ belong now to $P_{i+1}^{\prime}$ or $P_{i-1}^{\prime}$. The rest of the paths of $F^{\prime}$ only change in the length of some of the segments (see Figure 4).
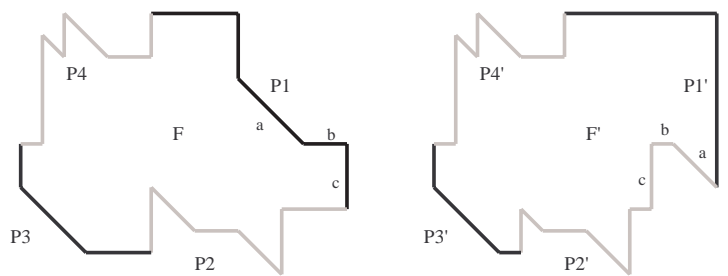

Fig. 4. How to restructure a face through the path $P_{1}$.

In the segment representation, the change of the face $F$ to $F^{\prime}$ produces some changes in the faces adjacent to $F$ along the path $P_{i}$, and we obtain another segment representation with convex faces as in the proof of Lemma 1 (see Figure 5). When we change the drawing in the manner described above, we say that the face $F$ is restructured through the path $P_{i}$.

We need at this point some new definitions. Given a 3-colored plane graph $G$ with colors $\{h, o, v\}$, a path $P=\left\{u_{1}, \ldots, u_{n}\right\}$ of $G$ is rare if it verifies one of the following conditions: 

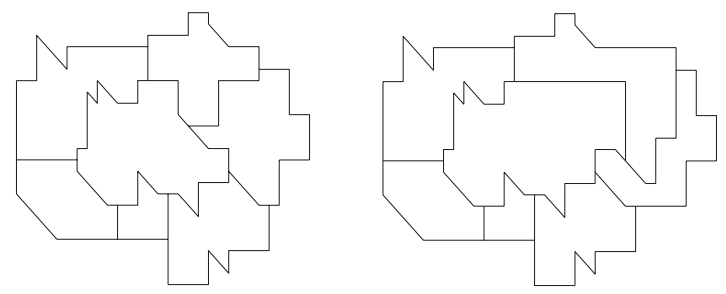

Fig. 5. How to restructure the faces adjacents to the restructured face $F$.

Case 1. $u_{1}$ is colored as $h, u_{2}$ as $o$, and $u_{n}$ as $v$.

Case 2. $u_{1}$ is colored as $v, u_{n-1}$ as $h$, and $u_{n}$ as $o$.

Case 3. $u_{1}$ is colored as $h, u_{2}$ as $o, u_{n-1}$ as $h$, and $u_{n}$ as $o$.

Case 4. $u_{1}$ is colored as $o, u_{2}$ as $v, u_{n-1}$ as $v$, and $u_{n}$ as $h$.

A face of a graph is called rare if there exists a path $P_{i}$, with $i=1, \ldots, 4$, containing a rare subpath. We say that a rare face is repaired if we subdivide its rare path with a new vertex colored as $v$ between $u_{1}$ and $u_{2}$ in the first and the third case, and between $u_{n-1}$ and $u_{n}$ in the second case, or a new vertex colored as $h$ between $u_{1}$ and $u_{2}$ in the fourth case.

On the other hand, notice that the vertical segments (except the last segment of $P_{1}$ and the last of $P_{3}$ ) can be extended to the interior of a convex face $F$, the horizontal segments too if they are in $P_{1}$ or $P_{3}$ (except the first of $P_{1}$ and the first of $P_{3}$ ), and the oblique segments if they are in $P_{2}$ or $P_{4}$ (except the first one of $P_{1}$ and $P_{3}$, and the last one of $P_{1}$ and $P_{3}$ ). Moreover, the horizontal segments in $P_{2}$ or $P_{4}$ can be extended to the interior of $F$ if there is a vertical segment to the right or an oblique segment to the left of them; and the oblique segments in $P_{1}$ or $P_{3}$ can be extended to the interior of $F$ if there is a horizontal segment to the right or a vertical to the left of them (see Figure 21). So, restructuring the paths and applying Lemma 1 we can transform the face $F$ in such a way that these transformations allow us to extend two segments inside $F$ to produce a new adjacency between them.

In the following lemma, when we say that two segments $s$ and $t$ can be joined by a path length $k$, it means a path $s, s_{1}, s_{2}, \ldots, s_{k}, t$.

Lemma 2. Let $I_{G}$ be a segment representation of a plane graph $G$ and let $s_{1}$ and $s_{2}$ be two distinct segments in a convex non-rare face $F$. Then $I_{G}$ can be transformed into another segment representation $I_{G}^{\prime}$ satisfying

1. $I_{G}$ and $I_{G}^{\prime}$ have the same faces and face boundaries as $G$, and the faces of $I_{G}^{\prime}$ are convex;

2. $s_{1}$ can be joined to $s_{2}$ by a segment path of length $k$, for all $k>0$, or directly if $s_{1}$ and $s_{2}$ have not the same direction inside the convex face $F^{\prime}$, corresponding to $F$. 
Proof. The proof falls naturally into two cases: the segments have the same direction, or they do not. In both cases, we have divided the proof in a sequence of subcases.

Case 1: $s_{1}$ and $s_{2}$ have not the same direction.

In this case, we can suppose that $s_{1}$ is vertical and $s_{2}$ is horizontal (similar arguments apply to the other configurations on the directions of the segments $s_{1}$ and $s_{2}$ ). The subcases are the following:

1. $s_{1}$ and $s_{2}$ are in the same path $P_{i}$.

a) $s_{1}$ and $s_{2}$ are in $P_{2}$ (respectively in $P_{4}$ ).

i. $s_{1}$ appears (clockwise) before $s_{2}$. If an oblique segment precedes $s_{2}$, by Lemma 1 the segments can be joined directly. Otherwise, the face must be restructured transforming $P_{2}$ into $P_{3}^{\prime}$ (respectively, $P_{4}$ into $\left.P_{1}^{\prime}\right)$.

ii. $s_{2}$ appears (clockwise) before $s_{1}$. Since the face is not rare, $s_{2}$ precedes a vertical segment. Then, by Lemma 1] the segments can be joined directly.

b) $s_{1}$ and $s_{2}$ are in $P_{1}$ (respectively in $P_{3}$ ). If $s_{1}$ appears (clockwise) before $s_{2}$, by Lemma 1 the segments can be joined directly. If $s_{2}$ appears (clockwise) before $s_{1}$, the face must be restructured transforming $P_{1}$ into $P_{2}^{\prime}$ (respectively, $P_{3}$ into $P_{4}^{\prime}$ ).

2. $s_{1}$ and $s_{2}$ are in different paths. If $s_{2}$ is in $P_{2}$ (respectively in $P_{4}$ ) and it precedes an oblique segment and $s_{1}$ is in $P_{3}$ (respectively $P_{1}$ ), the face must be restructured transforming $P_{2}$ into $P_{1}^{\prime}$ (respectively, $P_{4}$ into $P_{3}^{\prime}$ ). Otherwise, by Lemma 1 the segments can be joined directly.

Case 2: $s_{1}$ and $s_{2}$ have the same direction.

In this case, it suffices to join the segments by a path of length 1 , because this path can be substituted by any other of length greater than 1. Again there are several subcases:

1. Both are vertical segments. In this case, the segments can be joined by a path of length 1 directly using Lemma 1 .

2. Both are horizontal segments. If the segments are in $P_{2}$ (respectively in $P_{4}$ ) and the first one precedes an oblique segment, and a vertical segment precedes the second one, then the face must be restructured transforming $P_{2}$ into $P_{3}^{\prime}$ (respectively, $P_{4}$ into $P_{1}^{\prime}$ ). Otherwise, using Lemma 1 the segments can be joined directly.

3. Both are oblique segments. If the segments are in $P_{1}$ (respectively in $P_{3}$ ), the first one precedes a vertical segment, and a horizontal segment precedes the second one, then the face must be restructured transforming $P_{1}$ into $P_{2}^{\prime}$ (respectively, $P_{3}$ into $\left.P_{4}^{\prime}\right)$. Otherwise, they can be joined directly by Lemma 1

Now we prove:

Lemma 3. Any triangle-free plane graph which is a subdivision of a 3-connected graph can be represented by segments in three directions. 
Proof. As $G$ is a triangle-free plane graph, in virtue of Grötzsch's Theorem [9], $G$ admits a 3-coloring with the colors $h, v$ and $o$ (horizontal, vertical and oblique, respectively). Since $G$ is a subdivision of a 3 -connected graph, we can find a vertex, not in the outer face of $G$, connected by three disjoint paths to three vertices in the outer face. These paths and the outer face determine a graph $K$, which is a subdivision of $K_{4}$.

Using Barnette's results in [1] it is possible to build a sequence of graphs $G_{1}, \ldots, G_{n}$ and a sequence of paths $Q_{1}, \ldots, Q_{n}$ such that $G_{1}=G, G_{i}$ is a subdivision of a 3-connected plane graph, $G_{i}$ is obtained from $G_{i-1}$ by deleting the path $Q_{i-1}$, and the graph obtained from $G_{n}$ deleting $Q_{n}$ is $K$.

When the path $Q_{i}$ is deleted, a new face $F$ appears in $G_{i+1}$ as the union of two faces in $G_{i}$. The boundary of $F$ can be divided into two paths; on the one hand the path beginning in the first vertex of $Q_{i}$ and ending in the last one and, on the other hand, the rest of the boundary. If one of them is rare, we must repair $F$ subdividing an edge with a new vertex (that we label as a repaired vertex), to make the face $F$ non-rare. So, we can construct another sequence $G_{1}^{\prime}, \ldots, G_{n}^{\prime}$ where $G_{i}^{\prime}$ is $G_{i}$ with a new repaired vertex, if necessary.

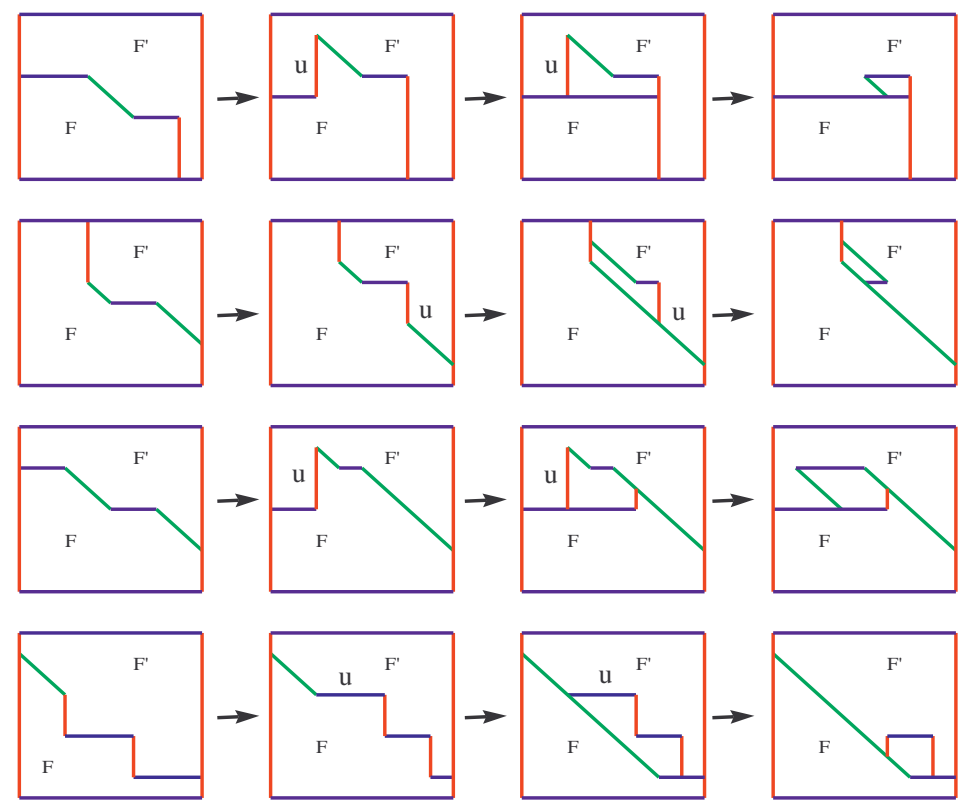

Fig. 6. How to remove the repaired vertex $u$ in the four cases.

It is easy to give a segment representation of $K^{\prime}$ with all its faces convex. It suffices to represent convexly the outer face of $K^{\prime}$ and proceed according to the 
above remark. Using Lemma 2 it is possible to add the path $Q_{i}$ to the segment representation of $G_{i+1}^{\prime}$ to obtain a convex face representation of $G_{i}^{\prime}$. In order to obtain a segment representation of the graph $G$, we must remove the repaired vertices. These vertices cannot be removed directly, so it is necessary to modify the representation. The four possible cases are illustrated in Figure 6 .

Notice that we could not remove the repaired vertices if the rare path would have exactly three segments $u_{1}, u_{2}$ and $u_{3}$, but this case it is not possible because joining $u_{1}$ with $u_{3}$ it will be form a triangle, and $G$ was a triangle-free graph.

We can now formulate our main result as follows:

Theorem 1. Every triangle-free planar graph is the intersection graph of a set of segments in three directions.

Proof. Let $G$ be a triangle-free plane graph. Since $G$ has no triangles, we can obtain a 3-coloring of $G$ using Grötszch's Theorem [9]. The colors will be labeled as $h, v$ and $o$ (horizontal, vertical and oblique, respectively). We can build a new triangle-free plane graph $G_{1}$, subdivision of a 3-connected graph, which contains $G$ as a subgraph, adding new vertices and edges joining the blocks of $G$, possibly subdividing some edges of the blocks of $G$ using new vertices. If this is the case, these vertices are labeled as dummy vertices. When an added edge produces a triangle, we subdivide it, and when a new edge joins two vertices with the same color, we subdivide it too. We call these new vertices and edges virtual. All the new vertices (dummy and virtual) can be colored so that the 3 -coloring is preserved .

By Lemma $3 . G_{1}$ admits a segment representation. Out of this segment representation we must remove all the vertices and edges added.

A virtual edge (or a path built with virtual edges and vertices) in the segment representation of $G_{1}$ is an adjacency between two segments (or a virtual path joining two segments). It suffices to break this adjacency and to shorten these segments (note that the segments do not cross, they only contact). The dummy vertices are removed as the repaired vertices in Lemma 3.

\section{Concluding Remarks}

The hypothesis that the graph has no triangles can be relaxed in some cases. No doubt there exist planar graphs with triangles that admits segment representations (see Figure [1). The problem lies in the fact that we have followed a constructive proof to obtain a segment representation using the convex faces representation of a subdivision of a 3-connected graph. This construction would not be possible in general if the graph contains triangles, because if we fix the three directions of the segments we can observe that the graph in Figure[7]cannot be represented by non-crossing segments.

In this example we see that a segment representation contains faces that do not correspond to faces of the embedding of the graph. So, the topology of the embedding of the plane graph cannot be preserved. This problem admits a 

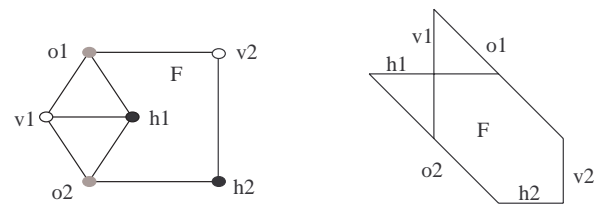

Fig. 7. Two segments must cross.

partial solution as follows. A 3-coloring of a plane graph $G$ with colors $\{h, v, o\}$ is good if all the triangles of $G$ are colored, in clockwise order, as $h-v-o$.

The proof of Theorem 1 can be adapted yielding the following result:

Theorem 2. Let $G$ be a 3-colored plane graph. The coloring is good if and only if exists a segment representation $I_{G}$ verifying that the faces of $G$ correspond to faces of $I_{G}$, and the boundaries of the faces are preserved.

\section{References}

1. D.W. Barnette, "On Steinitz's theorem concerning convex 3-polytopes and on some properties of planar graphs", The many facets of graph theory. Lectures Notes in Mathematics Vol. 110, Springer, Berlin, pp. 27-39, 1969.

2. I. Ben-Arroyo Hartman, I. Newman and R. Ziv, "On grid intersection graphs", Discrete Math. Vol. 87 , pp. 41-52, 1991.

3. H. de Fraysseix, P.Osona de Mendez and J. Pach, "Representation of planar graphs by segments", Colloquia Mathematica Societatis János Bolyai, Intuitive Geometry, Szeged (Hungary), 1991.

4. M.C. Golumbic, "Algorithmic Graph Theory and Perfect Graphs", Academic Press, 1980.

5. J. Kratochvíl, "A special planar satisfiability problem and a consequence of its NP-completeness", Discrete Applied Math., Vol. 52, pp. 233-252, 1994.

6. W. Naji, "Reconnaisance des graphes de cordes", Discrete Math. Vol. 54, pp. 329337, 1985.

7. E.R. Scheinerman, "Intersection classes and multiple intersection parameters of graphs", Ph D. thesis, Princeton University, 1984.

8. J. Spinrad, "Recognition of circle graphs", J. of Algorithms, Vol. 16, pp. 264-282, 1994.

9. C. Thomassen, "Grötzsch's 3-colour theorem and its counterparts for the torus and the projective plane", J. Comb. Theory B Vol. 62, pp. 268-279, 1994. 